\title{
Ganhos esperados com a seleção a partir da seleção simultânea de diferentes caracteres quantitativos em progênies de Eucalyptus camaldulensis DEHNH
}

\section{Expected gains with selection from the simultaneous selection of different quantitative traits in progenies of Eucalyptus camaldulensis DEHNH}

\author{
William de Medeiros Silva ${ }^{1 *}$, Mario Luiz Teixeira de Moraes ${ }^{2}$, \\ Janete Motta da Silva Gonzaga², Silvelise Pupin² e Bruno Ettore Pavan ${ }^{2}$
}

\begin{abstract}
RESUMO
Este trabalho teve como objetivo comparar os ganhos obtidos com três índices seleção e seleção direta e indireta aplicados aos valores genéticos individuais em testes de progênies de $E$. camaldulensis, aos seis anos de idade, para as caracteres forma de fuste, diâmetro a altura do peito e altura total das árvores. Os experimentos foram implantados em delineamento de blocos completos casualizados, com 133 progênies e espaçamento de $3,0 \mathrm{~m} \times 1,8 \mathrm{~m}$. O EXP 1 foi composto por parcelas experimentais de 5 plantas e quatro repetições e no EXP 2 utilizou-se uma planta por parcela e 20 repetições. As estimativas de componentes de variância e parâmetros genéticos foram obtidas utilizando o método REML/BLUP. A seleção dos indivíduos foi realizada com base nos valores genéticos aditivos individuais, por meio de seleção direta e indireta dos caracteres e paralelamente por meio dos índices de seleção da soma de postos, dos ganhos genéticos esperados e do somatório Z. Os índices de seleção, em comparação à seleção direta e indireta, proporcionaram ganhos mais bem distribuidos entre os caracteres. $O$ índice da soma de postos e índice $Z$ foram os que proporcionaram maiores ganhos genéticos com seleção simultânea no EXP1 $(12,74 \%)$ e EXP 2 (20,82\%), respectivamente. Os índices de seleção $Z$ e soma de postos foram os que apresentaram resultados mais semelhantes entre os métodos de seleção testados na seleção individual pelo valor genético aditivo. O EXP 2, por apresentar maiores valores para herdabilidade estimada, proporcionou maiores ganhos com a seleção.
\end{abstract}

Palavras-chave: Índice de seleção, Seleção direta, Teste de progênies.

\begin{abstract}
This study aimed to compare the gains obtained with three selection indices and direct and indirect selection applied to the individual genetic values in progeny tests of $E$. camaldulensis, at the age of six; for shape of stem, diameter at chest height and total height of the trees. The tests were implanted in a randomized complete block design, with 133 progenies and $3.0 \mathrm{~m} \times 1.8 \mathrm{~m}$ spacing. Trial 1 (EXP 1) was composed of experimental plots of 5 plants and four replications and in Trial 2 (EXP 2) single tree plot and 20 replicates were used. Estimates of variance components and genetic parameters were obtained using the REML/BLUP method. Selection of individuals was performed based on the individual additive genetic values, through direct and indirect selection of characters and in parallel through the indices of selection of the sum of ranks, expected genetic gains and the sum of $Z$. The selection indices, in comparison to direct and indirect selection, provide more balanced gains. The index of the sum of ranks and index $Z$ were those that provided greater genetic gains with simultaneous selection in EXP1 (12,74\%) and EXP 2 (20,82\%), respectively. The selection index $Z$ and sum of ranks were those that presented the most similar results among the selection methods tested in the individual selection by the additive genetic value. EXP 2, because it presents higher values for estimated heritability, provided greater gains with selection.
\end{abstract}

Keywords: Selection index, Direct selection, Progeny test.

1. Faculdade de Ciências Agrárias e Veterinárias - FCAV, Universidade Estadual Paulista "Júlio de Mesquita Filho" - Unesp. Jaboticabal, SP, Brasil.

2. Faculdade de Engenharia do Campus de Ilha de Solteira - FEIS, Universidade Estadual Paulista "Júlio de Mesquita Filho" - Unesp. Ilha Solteira, SP, Brasil.

* Autor correspondente: will.medeirossilva@gmail.com 


\section{INTRODUÇÃO}

Nos trabalhos de melhoramento de espécies florestais, a obtenção de fenótipos superiores para seleção e recombinação de progênies e indivíduos é uma ferramenta de considerável importância. A seleção baseada em um ou poucos caracteres pode se mostrar inadequada por não levar a um produto final superior com relação a vários caracteres.

Na maioria dos programas de melhoramento genético de eucalipto o caráter foco é o volume, e apesar da alta correlação entre os caracteres diâmetro a altura do peito (DAP) e altura total (FARIA et al., 2013; MACEDO et al., 2013, ZANDONÁ et al., 2008), a mensuração indireta do volume a partir do DAP e altura podem acarretar desvios na seleção dos indivíduos já que os erros associados ao volume são consideravelmente superiores (ROCHA et al., 2007). Além dos caracteres de crescimento, outro importante que pode influenciar significativamente no valor do produto final das indústrias de eucalipto é a forma do fuste (SOUZA, 2013), de modo que a seleção baseada em um único caráter pode não atender as necessidades do melhorista, principalmente em etapas avançadas de seleção. Nesse sentido, a teoria de índices de seleção seria uma alternativa permitindo combinar as múltiplas informações contidas nas unidades experimentais, de modo a selecionar com base em um grupo de características (VAYEGO et al., 2014).

Os índices de seleção são técnicas multivariadas com as quais se cria um caráter adicional, teórico, resultante da combinação dos caracteres sobre os quais o melhorista deseja realizar a seleção simultânea (CRUZ; REGAZZI, 1994). O primeiro índice de seleção foi proposto por Smith (1936), que posteriormente foi adaptado por Hazel (1943), desde então, surgiram inúmeras alternativas de índices de seleção. Dentre os índices, o baseado nos ganhos desejados de Pesek e Baker (1969), que tem sido usado em trabalhos com espécies florestais (BHERING et al., 2012; MARTINS et al., 2003; PAULA et al., 2002), e o índice desenvolvido por Mulamba e Mock (1978), conhecido como índice de soma de postos, no qual não há necessidade de se estabelecer pesos econômicos e de se ter estimativas de variâncias e covariâncias genotípicas e fenotípicas. O índice da soma de postos tem sido usado satisfatoriamente na seleção de diversas culturas como cana-de-açúcar (PEDROZO et al., 2009), milho-pipoca (SANTOS et al., 2007), açaizeiro (TEIXEIRA et al., 2012), pinus (MISSIO et al., 2004) e eucalipto (MARTINS et al., 2006).

Uma alternativa mais recente de índice de seleção é o índice Z proposto por Nunes et al. (2005), também conhecido como "bola cheia ou bola murcha". Este índice utiliza a padronização dos caracteres associados à uma possível visualização gráfica e possibilita notar em que caracteres a progênie e/ou indivíduo tem fenótipos favoráveis e em quais este é deficiente, além de permitir uma rápida e fácil identificação dos indivíduos de melhor performance (MENDES et al., 2009). Reis et al. (2015) utilizaram este índice pela primeira vez no setor florestal para duas espécies do gênero Eucalyptus, onde comparando-o com os índices clássico e soma de postos, e concluíram que o índice Z foi tão eficiente quanto os demais índices estudados.

No entanto, todos os índices foram testados para seleção de progênies, porém no melhoramento florestal a prática de seleção individual pelo valor genético do indivíduo é comum. Costa et al. (2015) testando métodos de seleção obtiveram ganhos superiores quando adotaram a seleção individual em progênies de E. camaldulensis, Freitas et al. (2009) encontraram melhores ganhos quando adotaram a seleção individual combinada para progênies de polinização aberta de híbridos de eucalipto. Entretanto, a utilização de índices de seleção aplicados aos valores genéticos dos indivíduos ainda é pouco utilizada, e sua eficiência não comprovada.

Nesse contexto, o objetivo do presente trabalho foi comparar os ganhos obtidos com três índices seleção e seleção direta e indireta aplicados aos valores genotípicos individuais para caracteres de crescimento (DAP e altura das árvores) e forma de fuste em dois testes de progênies de E.camaldulensis.

\section{MATERIAL E MÉTODOS}

Sementes de 250 progênies de polinização livre (meia irmãs) selecionadas em uma população base originária de 25 matrizes de E.camaldulensis procedentes da Austrália foram usadas para estabelecer dois testes de progênies (SILVA, 2010). Os testes foram instalados em outubro de 2008 na Fazenda de Ensino, Pesquisa e Extensão, da Faculdade de Engenharia de Ilha Solteira (FEIS/UNESP) localizada no município de Selvíria - MS, a $20^{\circ} 20^{\prime} 25^{\prime \prime}$ de latitude Sul e $51^{\circ} 24^{\prime} 33^{\prime \prime}$ de longitude Oeste, a 
Silva et al. - Ganhos esperados com a seleção a partir da seleção simultânea de diferentes caracteres quantitativos em progênies de Eucalyptus camaldulensis DEHNH

$371 \mathrm{~m}$ acima do nível do mar. O delineamento experimental utilizado, em ambos os testes, foi o de blocos completos casualizados, com 133 tratamentos (progênies) e espaçamento de 3,0 m x 1,8 m. O Experimento 1 (EXP1) foi composto por parcelas experimentais de 5 plantas e quatro repetições, e o Experimento 2 (EXP2) composto por uma planta por parcela e 20 repetições.

Aos seis anos de idade foram mensurados os seguintes caracteres de crescimento: circunferência a altura do peito (CAP, $\mathrm{cm}$ ) e altura total das árvores (ALT, m). A partir do caráter CAP obteve-se o diâmetro a altura do peito (DAP, $\mathrm{cm}$ ).

Paralelamente, avaliou-se a forma de fuste das árvores, com base em uma escala de notas de 1 (muito bifurcada) a 7 (sem bifurcações) para bifurcação das árvores, e outra escala para tortuosidade das árvores com notas de 1 (tortuosidade acentuada) a 7 (sem tortuosidade). As notas dadas para bifurcação e tortuosidade das árvores foram somadas gerando notas de 2 a 14 para o caráter forma de fuste.

As estimativas dos componentes de variância e parâmetros genéticos foram preditas para cada caráter por meio de análises individuais para os dois testes de progênies, utilizando o do software genético-estatístico SELEGEN, desenvolvido por Resende (2007) por meio do procedimento REML/BLUP (máxima verossimilhança restrita/melhor predição linear não viciada).

Para a análise do EXP 1 assumiu-se as progênies como sendo de meios-irmãos com várias observações

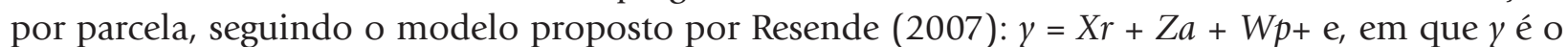
vetor de dados, $r$ é o vetor dos efeitos de repetição (assumidos como fixos) somados à média geral, $a$ é o vetor dos efeitos genéticos aditivos individuais (aleatórios), p é o vetor dos efeitos de parcelas (aleatórios), e é o vetor de erros ou resíduos (aleatórios). As letras maiúsculas representam as matrizes de incidência para os referidos efeitos. Na análise do EXP 2 assumiu-se as progênies como sendo de meias-irmãs com uma observação por parcela, seguindo o modelo proposto por Resende (2007): $y=X r+Z a+e$, que é semelhante ao primeiro modelo porém sem o vetor $p$.

Os parâmetros genéticos e componentes de variância estimadosforam: herdabilidade individual no sentido restrito $\left(h_{a}^{2}\right)$, coeficiente de variação genética aditiva individual $\left(\mathrm{CV}_{g i} \%\right)$, coeficiente de variação residual $\left(\mathrm{CV}_{e} \%\right)$, e média geral.

Para a seleção individual das árvores foram adotados quatro critérios de seleção realizados com base nos valores genéticos aditivos: seleção direta sobre cada um dos caracteres avaliados, na qual avaliou-se também a consequente mudança nos demais caracteres não selecionados diretamente; seleção com base em Soma de Postos ou "ranks", proposta por Mulamba e Mock (1978). Esse critério classifica as árvores em relação a cada um dos caracteres, em ordem favorável ao melhoramento, e posteriormente, soma-se as ordens de cada genótipo referente a cada caráter, resultando uma medida adicional tomada como índice de seleção (CRUZ; REGAZZI, 1994). Esse método é descrito da seguinte maneira: $\mathrm{I}=\mathrm{r} 1+\mathrm{r} 2+\ldots+\mathrm{rn}$, sendo que I é o valor do índice para determinado indivíduo; rj é a classificação (ou "rank") de um indivíduo em relação ao j-ésimo caráter; n é o número de caracteres considerado no índice; seleção mediante o índice dos ganhos desejados (PESEK; BAKER, 1969) no qual se considera o desvio-padrão genético entre os indivíduos, relativo a cada caráter, como vetor de ganhos desejados. Este índice foi realizado com auxílio do programa computacional GENES (CRUZ, 2008), considerando a seguinte expressão: $b=G^{-1} \Delta g_{d}$, sendo $\Delta g_{d}$ o vetor de ganhos desejados estabelecido pelo melhorista, $G$ é a matriz de variâncias e co-variâncias genéticas entre os caracteres e $b$ é o vetor $\mathrm{n} \times 1$ de coeficientes do índice; e seleção pelo índice somatório $\mathrm{Z}$ que foi estimada pelo seguinte estimador: $Z_{i}=\frac{a_{i}}{3}$, em que, $Z$ é o valor do caráter padronizado correspondente ao indivíduo $i$, a é o valor genético aditivo do caráter considerado do indivíduo $i$, e $s$ é o desvio padrão da média. A intensidade de seleção praticada entre as árvores foi de 33\%, que corresponde a 860 árvores selecionadas. Essa intensidade de seleção foi adotada visando a população com mais indivíduos remanescentes e uma ampla base genética.

Os ganhos obtidos com a seleção direta e indireta e com os índices de seleção foram calculados tanto em valores absolutos quanto em porcentagem a partir da média do valor genético aditivo individual das árvores selecionadas em cada situação.

\section{RESULTADOS E DISCUSSÃO}

Os coeficientes de variação residual encontrados apresentaram valores que variaram de 11,44 (ALT) a 14,34 (forma de fuste) no EXP 1 e de 22,56 (ALT) a 27,08 (DAP) no EXP 2 (Tabela 1). Estes valores estão próximos dos encontrados por Costa et al. (2015) e abaixo dos encontrados por Moraes et al. (2007) para testes de progênies de E. camaldulensis. 
TABELA 1: Etimativas de herdabilidade individual no sentido restrito $\left(h_{a}^{2}\right)$, coeficiente de variação genética aditiva individual $\left(C V_{g i} \%\right)$, coeficiente de variação residual $\left(C V_{e} \%\right)$, coeficiente de variação relativa $\left(C V_{r} \%\right)$ e Média do experimento (MG) para forma do fuste, diâmetro a altura do peito (DAP) e altura total da árvore (ALT) para os testes de progênies de E. camaldulensis com cinco plantas por parcelas (EXP 1) e uma planta por parcela (EXP 2).

TABLE 1: Estimate of individual heritability in the narrow sense $\left(h_{a}^{2}\right)$, coefficient of individual additive genetic variation $\left(\mathrm{CV}_{g i} \%\right)$, coefficient of residual variation $\left(\mathrm{CV}_{e} \%\right)$, coefficient of relative variation $\left(\mathrm{CV}_{r} \%\right)$ e Mean of experiment (MG) for stem shape, diameter at breast height (DAP) and total height of the tree (ALT) for the progenies of E. camaldulensis with five plants per plot (EXP 1) and single tree plot (EXP 2).

\begin{tabular}{ccccccc}
\hline Componentes & \multicolumn{2}{c}{ FORMA FUSTE } & \multicolumn{2}{c}{ DAP } & \multicolumn{2}{c}{ ALT } \\
\cline { 2 - 7 } de Variância & EXP 1 & EXP 2 & EXP 1 & EXP 2 & EXP 1 & EXP 2 \\
\hline$h_{a}^{2}$ & $0,14 \pm 0,04$ & $0,16 \pm 0,04$ & $0,16 \pm 0,04$ & $0,34 \pm 0,06$ & $0,19 \pm 0,04$ & $0,32 \pm 0,06$ \\
$C V_{e} \%$ & 14,35 & 23,15 & 11,97 & 27,08 & 11,45 & 22,56 \\
$C V_{r} \%$ & 0,742 & 0,411 & 0,921 & 0,61 & 0,941 & 0,596 \\
$C V_{g i} \%$ & 10,65 & 9,53 & 11,03 & 16,52 & 10,77 & 13,45 \\
MG & 8,62 & 9,00 & 12,12 & 12,3 & 17,1 & 18,19 \\
\hline
\end{tabular}

O coeficiente de variação genética aditiva individual $\left(\mathrm{CV}_{g i} \%\right)$, que expressaem porcentagem da média geral a quantidade de variação genética herdável existente entre indivíduos, variou de 10,65\% (forma de fuste) a 11,03\% (DAP) e 9,53\% (forma de fuste) a 16,52\% (DAP) no EXP 1 e EXP 2, respectivamente (Tabela 1). Segundo Sebbenn et al. (1998) coeficientes de variação acima de 7\% são considerados altos, portanto, nesse caso, há a possibilidade de encontrar indivíduos superiores dentro de cada progênie que proporcionarão ganhos com seleção. Estes valores encontrados são condizentes com os valores obtidos por Costa et al. (2015) ao avaliarem as mesmas progênies de E. camaldulensisavaliadas neste trabalho no município de Santo Antônio do Leverger - MS.

O coeficiente de variação relativa foi maior no EXP 1, mantendo-se próximo à 1 nos três caracteres (Tabela 1). Segundo Vencovsky (1987), quanto mais próximo de 1 forem as relações $C V_{g i} / C V_{e^{\prime}}$ melhores as chances de ganhos com a aplicação de seleção, sugerindo que a característica pode ser trabalhada facilmente no melhoramento.

Para Resende (1995), herdabilidades de 0,15 a 0,50 são consideradas medianas, e todos os valores encontrados para esse parâmetro neste trabalho se mantiveram dentro dessa faixa, variando

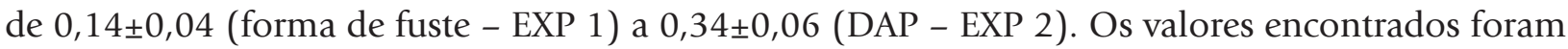
superiores aos encontrados por Costa et al. (2015) em um teste com as mesmas progênies em um outro sítio e de E. tereticornis (MACEDO et al., 2013). A herdabilidade estimada no sentido restrito $\left(h_{a}^{2}\right)$ é a mais útil entre as herdabilidades, pois quantifica a importância relativa da proporção aditiva da variância genética que pode ser transmitida para a próxima geração (BORÉM; MIRANDA 2009).

Na seleção direta dos caracteres (Tabela 2), os maiores ganhos obtidos no EXP 1 ocorreram para o caráter ALT $(11,78 \%)$ e no EXP 2 para o caráter DAP $(11,67 \%)$. Já a seleção direta do caráter forma de fuste foi a que proporcionou os menores ganhos individuais tanto no EXP 1 (5,17\%) como no EXP 2 (5,10\%), o que já era esperado já que o caráter apresentou os menores coeficientes de variação genética. (Tabela 1).

TABELA 2: Estimativas dos progressos genéticos com a seleção direta e indireta, entre progênies de meios-irmãos de Eucalyptuscamaldulensis, aos 6 anos de idade, em Selvíria, MS, onde: ALT = altura total das árvores; DAP = diâmetro a altura do peito; FF = forma de fuste.

TABLE 2: Estimates of genetic progress with direct and indirect selection, between progenies of half-brothers of Eucalyptus camaldulensis, at 6 years of age, in Selvíria, MS; where: ALT = total height of trees; $\mathrm{DAP}=$ diameter at breast height; FF = bole shape.

\begin{tabular}{cccccc}
\hline \multirow{2}{*}{ EXPERIMENTO } & CÁRATER & \multicolumn{4}{c}{ RESPOSTA ESPERADA } \\
\cline { 3 - 6 } & SELECIONADO & ALT & DAP & FF & TOTAIS (\%) \\
\hline \multirow{2}{*}{ EXP 1 } & ALT & $1,016(11,78 \%)$ & $0,533(4,39 \%)$ & $0,099(1,14 \%)$ & $17,31 \%$ \\
& DAP & $0,732(4,28 \%)$ & $0,721(5,94 \%)$ & $0,032(0,37 \%)$ & $10,59 \%$ \\
& FF & $0,207(1,21 \%)$ & $0,068(0,56 \%)$ & $0,446(5,17 \%)$ & $6,94 \%$ \\
EXP 2 & ALT & $1,637(8,99 \%)$ & $1,185(9,63 \%)$ & $0,074(0,81 \%)$ & $19,43 \%$ \\
& DAP & $1,376(7,56 \%)$ & $1,436(11,67 \%)$ & $0,027(0,29 \%)$ & $19,52 \%$ \\
& FF & $0,161(0,88 \%)$ & $-0,0007(-0,005 \%)$ & $0,463(5,10 \%)$ & $5,97 \%$ \\
\hline
\end{tabular}


Silva et al. - Ganhos esperados com a seleção a partir da seleção simultânea de diferentes caracteres quantitativos em progênies de Eucalyptus camaldulensis DEHNH

Na seleção direta e indireta dos caracteres altura $(17,31 \%)$ e DAP $(19,52 \%)$ foram os que proporcionaram os maiores porcentagens de ganhos genéticos no EXP 1 e EXP 2, repectivamente.

De maneira geral, o EXP 2 proporcionou maiores ganhos totais para os caracteres de crescimento com a seleção direta quando comparado aos ganhos obtidos com esses caracteres no EXP 1. Já para o caráter forma de fuste o progresso genético esperado foi mais expressivo no EXP 1.

Os caracteres ALT e DAP proporcionaram os maiores progressos esperados indiretamente com a seleção direta sobre um dos caracteres nos dois experimentos (Tabela 2). Esses resultados devem-se às altas e positivas correlações genéticas e fenotípicas observadas entre os caracteres de crescimento (COSTA et al., 2012). Resultados semelhantes para esses dois caracteres foram encontrados por Martins et al. (2006) ao testarem a eficiência da seleção direta e indireta em Eucalyptusgrandis.

Os ganhos obtidos para o caráter forma de fuste pela seleção direta do mesmo foram superiores em relação à seleção indireta nos dois experimentos (Tabela 2). A forma do fuste não apresenta correlações positivas e significativas com os caracteres de crescimento. Dessa maneira, a seleção direta é mais indicada para esse caráter. A seleção direta na forma de fuste proporcionou valores negativos para ganhos com DAP no EXP 2.

De maneira geral, os progressos genéticos esperados com a seleção direta dos caracteres tiveram valores próximos aos obtidos com os índices de seleção (Tabela 3). No EXP 1, por exemplo, o maior ganho total obtido entre todas as estratégias de seleção foi com a seleção direta do caráter ALT $(17,31 \%)$. Entretanto, a distribuição dos ganhos entre os caracteres foi mais equilibrada com os índices de seleção do que com a seleção direta e indireta dos caracteres, e uma vez que tanto os caracteres de crescimento como a forma de fuste das árvores refletem no valor final do produto, um método de seleção que permita obter ganho em múltiplos caracteres é mais viável para o melhorista em etapas avançadas de seleção. Resultados semelhantes foram encontrados por Missio et al. (2004) ao estudarem progênies de Pinus caribaeaMorelet var. bahamensis.

TABELA 3: Estimativas dos progressos genéticos com três índices de seleção entre progênies de meios-irmãos de Eucalyptuscamaldulensis, aos 6 anos de idade, em Selvíria, MS, onde: ALT = altura total das árvores, $\mathrm{DAP}=$ diâmetro a altura do peito, $\mathrm{FF}=$ forma de fuste, $\mathrm{MM}=0$ índice das somas de postos, $\mathrm{PB}=$ índice dos ganhos desejados e $Z$ = índice somatório $Z$.

TABLE 3: Estimates of genetic progress with three selection indices between half-sib progenies of Eucalyptus camaldulensis, at 6 years of age, in Selvíria, MS; where: ALT = total height of trees; DAP = diameter at breast height; $\mathrm{FF}=$ bole shape, $\mathrm{MM}=$ index of sums of posts, $\mathrm{PB}=$ desired earnings index and $Z$ = summation index $Z$.

\begin{tabular}{ccrrrr}
\hline \multirow{2}{*}{ EXPERIMENTO } & \multirow{2}{*}{ ÍNDICE } & \multicolumn{3}{c}{ CARACTERES } & \multirow{2}{*}{ TOTAIS (\%) } \\
\cline { 2 - 5 } & ALT & DAP & FF & \\
\hline EXP 1 & MM & $0,885(5,17 \%)$ & $0,58(4,78 \%)$ & $0,241(2,79 \%)$ & $12,74 \%$ \\
& PB & $0,51(2,98 \%)$ & $0,587(4,84 \%)$ & $0,255(2,95 \%)$ & $10,77 \%$ \\
& $Z$ & $0,817(4,77 \%)$ & $0,518(4,27 \%)$ & $0,23(2,66 \%)$ & $11,76 \%$ \\
EXP 2 & MM & $1,491(8,19 \%)$ & $1,21(9,83 \%)$ & $0,21(2,31 \%)$ & $20,33 \%$ \\
& PB & $0,233(1,28 \%)$ & $0,136(1,10 \%)$ & $0,456(5,02 \%)$ & $7,40 \%$ \\
& Z & $1,548(8,51 \%)$ & $1,328(10,79 \%)$ & $0,138(1,52 \%)$ & $20,82 \%$ \\
\hline
\end{tabular}

No EXP 1 o índice da soma de postos foi o que propiciou maior progresso genético com a seleção (12,74\%), seguido pelo índice Z (11,76\%) e pelo índice dos ganhos desejados(10,77\%) (Tabela 3). Considerando que a distribuição dos ganhos entre os caracteres estudados no EXP 1 foi semelhante para os três índices, pode-se afirmar que o índice proposto por Mulamba e Mock (1978) foi o mais eficiente para a seleção simultânea de caracteres no EXP 1. Já no EXP 2, o índice Z foi o que proporcionou maiores ganhos com a seleção simultânea $(20,82 \%)$ seguido pelo índice da soma de postos (20,33\%) e pelo índice dos ganhos desejados (7,40\%). Sendo assim, o índice Z foi o mais eficiente no EXP 2. O índice dos ganhos desejados foi, portanto, o que proporcionou os menores progressos esperados com a seleção nos dois experimentos.

Comparando os ganhos totais obtidos com os três índices de seleção entre os dois experimentos, nota-se que para os índices da soma de postos e do somatório $\mathrm{Z}$ os ganhos obtidos no EXP 2 foram consideravelmente maiores do que os ganhos obtidos no EXP 1. Já para o índice dos ganhos desejados, os maiores ganhos totais foram obtidos no EXP 1. 
Com base nos ganhos obtidos com os três índices de seleção nos dois experimentos, pode-se afirmar que os índices da soma de postos e o índice somatório $\mathrm{Z}$ foram os mais concordantes na seleção dos indivíduos. Reis et al. (2015), também observaram boa concordância entre esses dois índices ao estudar estratégias de seleção simultânea em híbridos de E. grandise E. urophylla. No entanto, pela facilidade de obtenção e ganhos moderadamente mais equilibrados, o índice de soma postos pode ser considerado adequado para trabalhos de melhoramento florestal para caracteres estudados.

Os ganhos estimados nos dois experimentos demostram maior eficiência da seleção quando aplicadas em experimentos de uma planta por parcela, justificado pelas maiores herdabilidades. Scarpinati et al. (2009) também relatou maior eficiência de experimentos com muitas repetições e uma planta parcela em testes clonais de eucalipto.

\section{CONCLUSÕES}

Os índices de seleção proporcionaram ganhos genéticos mais bem distribuídos entre os caracteres estudados do que a seleção direta e indireta;

Os índices de seleção Z e soma de postos foram semelhantes e mais eficientes na seleção individual pelo valor genético aditivo do que os demais métodos testados, sendo que o índice de soma de postos pode ser considerado o mais adequado visto sua facilidade de obtenção e pelos ganhos estimados nos dois testes; $\mathrm{e}$

Experimento com uma planta por parcela proporciona os maiores progressos esperados com a seleção devido à maior herdabilidade estimada.

\section{AGRADECIMENTOS}

Os autores agradecem aos funcionários da Fazenda de Ensino, Pesquisa e Extensão, da Faculdade de Engenharia de Ilha Solteira, pela condução das áreas experimentais. Willian M. Silva agradece a Coordenação de Aperfeiçoamento de Pessoal de Nível Superior (CAPES) pela concessão de bolsa de estudo a nível de mestrado. Mario L. T. Moraes agradece a bolsa de produtividade em pesquisa apoiada pelo Conselho Nacional de Desenvolvimento Científico e Tecnológico (CNPq).

\section{REFERÊNCIAS BIBLIOGRÁFICAS}

BHERING, L. L.; LAVIOLA, B. G.; SALGADO, C. C.; SANCHEZ, C. F. B.; ROSADO, T. B.; ALVES, A. A. Geneticgains in physicnutusingselection indexes. Pesquisa Agropecuária Brasileira, Brasília, v. 47, p. 402-408, 2012.

BORÉM, A.; MIRANDA, G. V. Melhoramento de plantas. 5. ed. Viçosa: UFV, 2009. 529 p.

COSTA, R. B.; MARTINEZ, D. T.; SILVA, J. C.; ALMEIDA, B. C. Variabilidade e ganhos genéticos com diferentes métodos de seleção em progênies de Eucalyptus camaldulensis. Revista Ciências Agrarias, Belém, v. 58, n. 1, p. 69-74, 2015.

COSTA, R. B.; AZEVEDO, L. P. A.; MARTINEZ, D. T.; TSUKAMOTO FILHO, A. A.; FERNANDES, D. A.; OLIVEIRA, O. E.; RESENDE, M. D. V. Avaliação genética de Eucalyptus camaldulensis no Estado de Mato Grosso. Pesquisa Florestal Brasileira, Colombo, v. 32, n. 70, p. 165-173, 2012.

CRUZ, C. D. Programa GENES: biometria. Viçosa: Editora UFV, 2008. 278 p.

CRUZ, C. D.; REGAZZI, A. J. Modelos biométricos aplicados ao melhoramento genético. Viçosa: Imprensa universitária, 1994. 390 p.

FARIA, J. R.; SILVA, J. F.; NERIS, K. P.; LOPES, F. L. R.; SILVA, M. C.; LISBOA, E. S.; RODRIGUES, J.; CENTENO, A. J.; LOPES, F. M. Desenvolvimento de Eucalyptusurograndis no município de Corumbá-GO. Ensaios e Ciência: Ciências Biológicas Agrárias e da Saúde, Valinhos, v. 17, n. 2. p. 9-, 2013.

FREITAS, R. G. VASCONCELOS, E.S.; CRUZ, C. D.; ROSADO, A. M.; ROCHA, R. B.; TAKAM, L. K. Predição de ganhos genéticos em progênies de polinização aberta de Eucalyptus urograndis cultivadas em diferentes ambientes e submetidas a diferentes procedimentos de seleção. Revista Árvore, v. 33, p. 255-263, 2009.

HAZEL, L. N. The genetic basis for constructing selection indexes. Genetics, Austin, v. 28, p. 476-490, 1943. 
Silva et al. - Ganhos esperados com a seleção a partir da seleção simultânea de diferentes caracteres quantitativos em progênies de Eucalyptus camaldulensis DEHNH

MACEDO, H. R.; FREITAS, M. L. M.; MORAES, M. L. T. dE; ZANATA, M.; SEBBEN, A. M. Variação, herdabilidade e ganhos genéticos em progênies de Eucalyptustereticornisaos 25 anos de idade em Batatais-SP. Scientia Forestalis, Piracicaba, v. 41, n. 100, p. 533-540, dez. 2013.

MARTINS, I. S.; MARTINS, R. C. C.; PINHO, D. S. Alternativas de índices de seleção em uma população de EucalyptusgrandisHill exMaiden. Cerne, Lavras, v. 12, n. 3, p. 287-291, 2006.

MARTINS, I. S.; CRUZ, C. D.; REGAZZI, A. J.; PIRES, I. E.; MARTINS, R. C. C. Avaliação de critérios multivariados aplicados na seleção em EucayptusgrandisHill exMaiden. Floresta e Ambiente, Seropédica, v. 10, n.1, p. 38 47, 2003.

MENDES, F. F.; RAMALHO, M. A. P.; ABREU, Â. de F. B. Índice de seleção para escolha de populações segregantes do feijoeiro-comum. Pesquisa Agropecuária Brasileira, Brasília, v. 44, p. 1312-1318, 2009.

MISSIO, R. F.; CAMBUIM, J.; MORAES, M. L. T.; PAULA, R. C. Seleção simultânea de caracteres em progênies de Pinus caribaeaMorelet var. bahamensis. Scientia Forestalis, Piracicaba, n. 66, p. 161-168, 2004.

MORAES, M. A.; ZANATTO, A. C. S.; MORAES, E.; SEBBEBB, A. M.; FREITAS, M. L. M. Variação genética para caracteres silviculturais em progênies de polinização aberta de Eucalyptuscamaldulensisem. Revista Instituto Florestal Luiz Antonio, v. 19, n. 2, p. 113-118, 2007.

MULAMBA, N. N.; MOCK, J. J. Improvement of yield potential of the Eto Blanco maize (Zea mays L.) population by breeding for plant traits. Egyptian Journal of Genetics and Cytology, Alexandria, v. 7, p. 40-51, 1978.

NUNES, J. A. R.; RAMALHO, M. A. P.; ABREU, A. F. B. Graphical method in studies of adaptability and stability of cultivars. Annual Report of the Bean Cooperative, Fort Collins, East Lansing, v. 48, p. 182-183, 2005.

PAULA, R. C.; PIRES, I. E.; BORGES, R. D. C. G.; CRUZ, C. D. Predição de ganhos genéticos em melhoramento florestal. Pesquisa Agropecuária Brasileira, Brasília, v. 37, n. 2, p. 159-165, 2002.

PEDROZO, C. A.; BENITES, F. R. G.; BARbOSA, M. H. P.; RESENDE, M. D. V. de; SILVA, F.L. da. Eficiência de índices de seleção utilizando a metodologia REML/BLUP no melhoramento da cana-de-açúcar. Scientia Agraria, Curitiba, v. 10, n. 1, p. 31-36, 2009.

PESEK, J.; BAKER, R. J. Desired improvement in relation to selected indices. Canadian Journal of Plant Science, Ottawa, v. 49, p. 803-804, 1969.

REIS, C. A. F.; GONÇALVES, F. M. A.; RAMALHO, M. A. P.; ROSADO, A. M. Estratégias na seleção simultânea de vários caracteres no melhoramento do Eucalyptus. Ciência Florestal, Santa Maria, v. 25, n. 2, p. 457-467, 2015.

RESENDE, M. D. V. Selegen-reml/blup: sistema estatístico e seleção genética computadorizada via modelos lineares mistos. Colombo: Embrapa Florestas, 2007. 359 p.

RESENDE, M. D. V. Delineamento de experimentos de seleção para maximização da acurácia seletiva e do progresso genético. Revista árvore, Viçosa, v. 19, n. 4, p. 470-500, 1995.

ROCHA, M. G. B.; PIRES, I. E.; ROCHA, R. B.; XAVIER, A.; CRUZ, C. D. Seleção de genitores de EucalyptusgrandisE DE Eucalyptusurophyllapara produção de híbridos interespecíficos utilizando REML/ BLUP e informação de divergência genética. Revista Árvore, Viçosa, v. 31, n. 6, p. 977-987, 2007.

SANTOS, F. S.; AMARAL JÚNIOR, A. T. do; FREITAS JÚNIOR, S. P.; RANGEL, R. M.; PEREIRA, M. G. Predição de ganhos genéticos por índices de seleção na população de milho-pipoca unb-2u sob seleção recorrente. Bragantia, Campinas, v. 66, n. 3, p. 389-396, 2007.

SCARPINATI, E. A.; PERECIN, D.; PAULA, R. C. DE; VALENCISE, C. A.; PAVAN, B. E.; CANDIDO, L. S. Influência do modelo de análise estatística e da forma das parcelas experimentais na seleção de clones de Eucalyptusspp. Revista Árvore, Viçosa, v. 33, n. 4, p. 769-776, 2009.

SEBBENN, A. M.; SIQUEIRA, A. C. M. F.; KAGEYAMA, P. Y.; MACHADO, J. A. R. Parâmetros genéticos na conservação da cabreúva - Myroxylonperuiferum L.F. Allemão. Scientia Forestalis, Piracicaba, n. 53, p. 3138, 1998. 
SILVA, J. M. Avaliação da variabilidade genética em uma população base de Eucalyptus camaldulensis Dehnh. para fins de conservação e melhoramento genético. 2010. 153 p. Tese (Doutorado em Ciências Florestais) - Faculdade de Engenharia, Universidade Estadual Paulista, Ilha Solteira, 2010.

SMITH, H. F. A discriminant function for plant selection. Annals of Eugenics, London, v. 7, p. 240-250, 1936.

SOUZA, R. R. Estudo da forma do fuste de árvores de eucalipto em diferentes espaçamentos. 2013. 87 p. Dissertação (Mestrado em Ciência Florestal) - Universidade Federal dos Vales do Jequitinhonha e Mucuri, Diamantina, 2013.

TEIXEIRA, D. H. L.; OLIVEIRA, M. S. P; GONÇALVES, F. M. A.; NUNES, J. A. R. Índices de seleção no aprimoramento simultâneo dos componentes da produção de frutos em açaizeiro. Pesquisa Agropecuária Brasileira, Brasília, v. 47, n. 2, p. 237-243, 2012.

VAYEGO, S. A.; DIONELLO, N. J. L.; FIGUEIREDO, E. A. P. Seleção direta e indireta e de índices de seleção em linhagens de frango de corte. Semina: Ciências Agrárias, Londrina, v. 35, n. 4, p. 2107-2116, 2014.

VENCOVSKY, R. Herança quantitativa. In: PATERNIANI, E.; VIEGAS, G. P. (Eds.). Melhoramento e produção do milho. 2. ed. Campinas: Fundação Cargill, 1987. p. 137-214.

ZANDONÁ, D. F.; LINGNAU, C.; NAKAJIMA, N. Y. Varredura a Laser aerotransportado para estimativa de caracteresdendrométricas. Scientia Forestalis, Piracicaba, v. 36, n. 80, p. 295-306, 2008.

Recebido em: 26/07/2018

Aceito em: 20/03/2019 